\title{
STUDY OF UNSTEADY HYDRODYNAMIC EFFECTS IN THE STERN AREA OF RIVER CRUISERS IN SHALLOW WATER
}

\author{
IVAN SHEVCHUK* and NIKOLAI KORNEV \\ * Chair of Modeling and Simulation, University of Rostock \\ Albert-Einstein Str. 2, 18059, \\ Rostock, Germany \\ ivan.shevchuk@uni-rostock.de
}

Key words: Shallow water, hybrid RANS/LES, unsteady propeller loadings, vibration

\begin{abstract}
The main objective of the present paper is the assessment of the influence of shallow water conditions on the hydrodynamic exciters of ship hull vibration using hybrid RANS/LES methods. The following topics will be briefly discussed:
\end{abstract}

- Validation of hybrid methods for calculation of ship wake in shallow water

- Analysis of the influence of different factors on the nominal wake characteristics

- Assessment of thrust fluctuations and pressure pulses and their sensitivity to motion conditions

It will be shown, that the depth restriction may influence the periodic forces and moments acting on the ship stern and by these means intensify the vibration.

\section{INTRODUCTION}

One the most important factors for design of river cruise ships is the passenger comfort. However, experience shows, that ship motion in fairways with depth restriction often leads to a rise of strong vibration at the stern which impairs the habitability conditions. This forces ship designers to either avoid placement of passenger cabins in the stern area, or to use additional damping construction to suppress the vibration. Both measures result in increase of costs and reduction of ship economic efficiency. The development of technical measures for vibration reduction requires understanding of main physical mechanisms leading to amplification of parasitic oscillations.

The main reason of hydrodynamically excited vibration is the propeller, which in multiple ways affects the hull. First, the forces and moments, produced by the propeller may considerably fluctuate in time because of the wake nonuniformity and instationarity. Second, the propeller 
blades rotating in water create pressure pulses on the hull and by these means cause the oscillations of the hull surface. The pressure pulses due to cavitation usually play the major role for vibration excitation [9]. If cavitation is not present, the lift part is dominant.

In this work the modelling of cavitation and the structural response to the described unsteady effects will left out of the scope in order to keep the computational effort on the feasible level. Thus present work deals only with the hydrodynamic excitation forces, not including the cavitation effects. As an object for the study the generic geometry of the inland cruise vessel is considered.

URANS approach, widely used for industrial applications nowadays, can provide satisfactory results for many tasks like resistance prediction, ship motion in waves, open water propeller tests, etc. $[5,2]$. However, when it comes to the unsteady flows, URANS tends to smear the flow features in time and space [3]. This fact motivated the application of hybrid RANS/LES methods for the considered task.

\section{VALIDATION}

Before applying the computational methodology to the case of interest it was necessary to compare the performance of different hybrid methods for the similar conditions. For this purpose the PIV measurements of the wake of an inland ship (model scale 1:40) in shallow water, provided by the Duisburg Development Centre for Ship Technology and Transport Systems(DST) were used [6]. The considered conditions are summarized in Table 1 , where $B_{c}$ is the breadth of the towing tank, $U$ - model speed, $h$ - water depth, $T$ - ship draught, $F n_{h}=\frac{U}{\sqrt{g h}}$ - depth Froude number. The stern geometry can be seen in Figure 1.

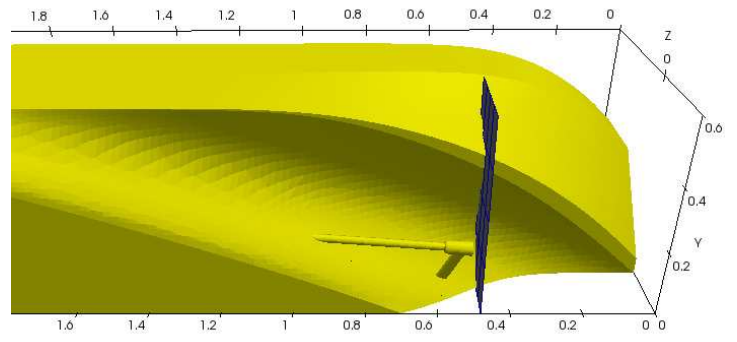

Figure 1: Stern geometry of M1926 model (left half). The plane, where the velocity field was measured in, is shown at $x=0.48$.

\begin{tabular}{llr}
\hline Par-r & & Value \\
\hline$B_{c}$ & {$[\mathrm{~m}]$} & 9.8 \\
$U$ & {$[\mathrm{~m} / \mathrm{s}]$} & 1.24 \\
$h$ & {$[\mathrm{~m}]$} & 0.5 \\
$T$ & {$[\mathrm{~m}]$} & 0.25 \\
$h / T$ & {$[-]$} & 2 \\
$F n$ & {$[-]$} & 0.12 \\
$F n_{h}$ & {$[-]$} & 0.56 \\
$R e$ & {$[-]$} & $1.36 \cdot 10^{7}$ \\
\hline
\end{tabular}

Table 1: Parameters of the model and the experimental conditions considered in the validation study.

Model dimensions were $L_{o a}=11 \mathrm{~m}, B=1.14 \mathrm{~m}, T=0.25 \mathrm{~m}$. No propeller or any other appendages except the shaft were present in experiment. The velocity was measured in the propeller plane.

The nominal wake $w=1-\left|\left\langle u_{x}\right\rangle\right| / U$ was calculated from the ensemble-averaged measured values of the longitudinal velocity and compared to the computed ones using contour plots (see Figure $5 \mathrm{~b}, \mathrm{c}$ and d) and one-dimensional plots along the circle $r / R=0.7$ in the propeller plane (see Figures 2a, b and c). 
Following turbulence models were used for validation: RANS $k-\omega$ SST, and SST-IDDES [4]. The description of the governing equations of the SST-IDDES model can be found in the corresponding papers and is omitted here. The averaged in time values of the wake were compared between different models for gradually refined hexa-dominant meshes (3M - 20M cells). For hybrid simulations the timestep was chosen from the condition $C o<1$ (for each cell), which is an essential constraint for scale-resolving simulations.

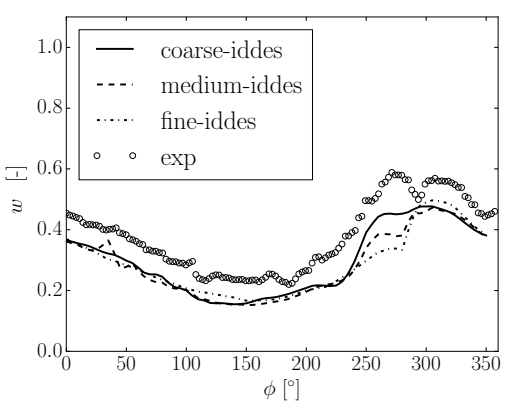

(a)

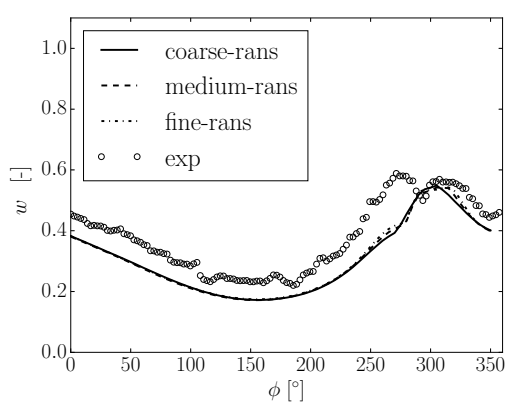

(b)

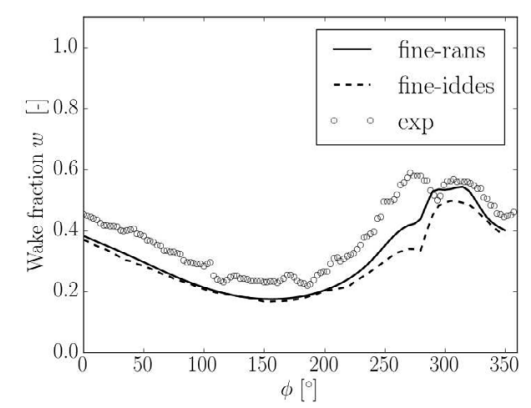

(c)

Figure 2: Comparison of the wake at $r / R=0.7$ obtained on different grids using different methods to experiment: (a) - SST-IDDES for different mesh resolutions, (b) - SST-RANS on the same meshes, (c) comparison of SST-RANS and SST-IDDES.

An important aspect of application of scale-resolving methods is the generation of the turbulent content. In the present work this was done using the modal turbulence generator, proposed in [1]. The turbulence generator in form of the momentum source was placed at a distance of $2(h-T)$ upstream from the end of the parallel middle body at the stern (see Figure 3 ). The statistics for the generator were taken from the preliminary RANS computation.

The SST-IDDES model and the synthetic turbulence generator were implemented by authors in OpenFOAM CFD toolkit, version 2.3.x and used in the solver pimpleFoam.

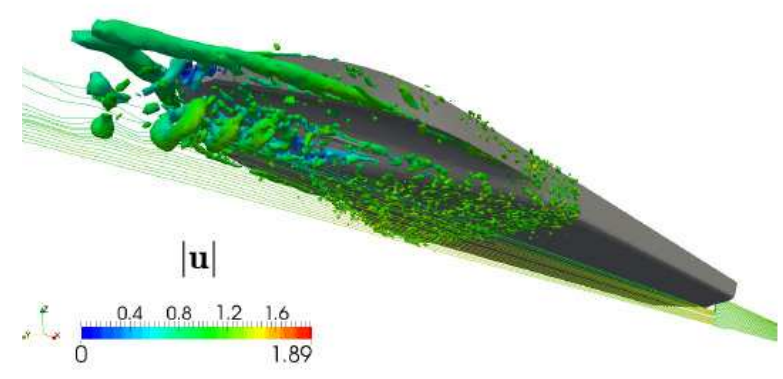

Figure 3: Vortical structures $\left(\lambda_{2}=-20\right)$ in the stern of M1926 predicted using SSTIDDES + synthetic turbulence generator. One can see, where the turbulent structures are added to the flow.

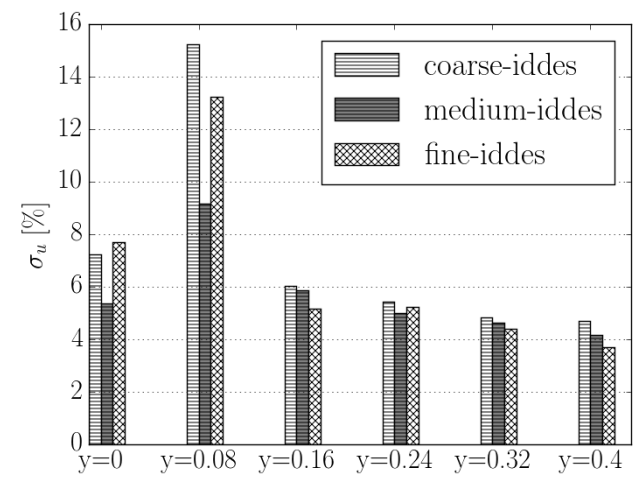

Figure 4: Dependence of the standard deviation of the longitudinal velocity $\sigma_{u}$ in percent of the inflow velocity for different mesh resolutions at the points, lying on in propeller plane at $\mathrm{z}=-0.23$.

From the plots, presented in Figures 2 and 5 one can see, that RANS $k-\omega$ SST model as 
well as SST-IDDES model predictions are in a good agreement with each other and with the experimental data. SST-RANS model turns out to give decent results for the considered flow. One can explain this by the absence of strong separations in the wake. However, as can be seen in Figure 5, the RANS model smears out the anchor-like form of the wake. The second problem, which one can mention - is the absence of the peak of the wake fraction at $\phi \approx 270^{\circ}$. The RANS solution seems to be insensitive to the mesh refinement.

The predictions, delivered by SST-IDDES model for the wake are of nearly the same quality as those, computed using SST-RANS. Moreover, in some regions the hybrid model is advantageous: it prevents the smearing of the wake form and is obviously able to capture the peak of the wake fraction at $\phi \approx 270^{\circ}$. Unfortunately, this is true only for the coarsest mesh. Mesh refinement in this case caused slight deterioration of the results in the region $225^{\circ}<\phi<300^{\circ}$, which is a surprising result. It indicates either the malfunctioning of the model shielding in response to mesh refinement or influence of mesh quality on the results (unstructured mesh was used).

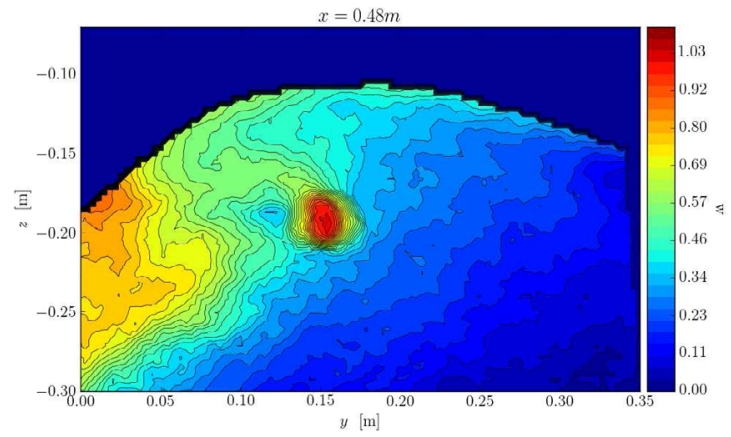

(a)

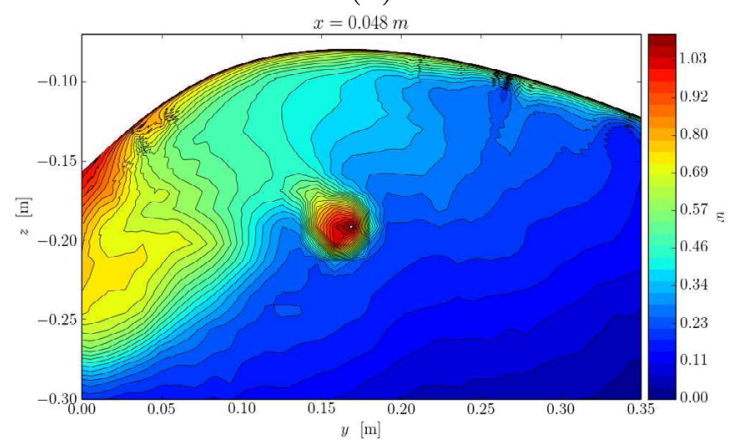

(c)

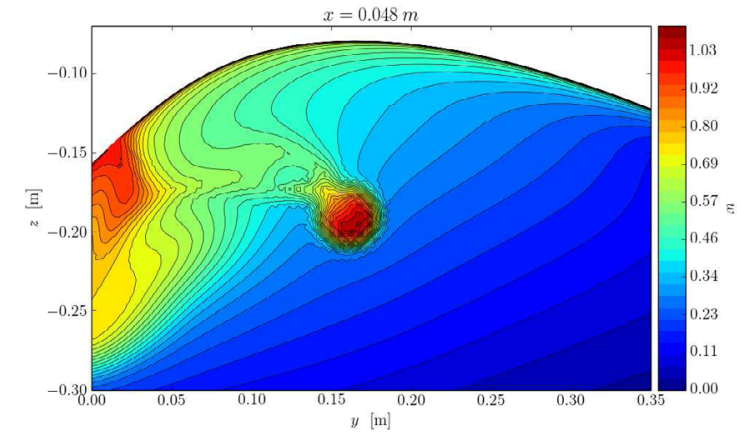

(b)

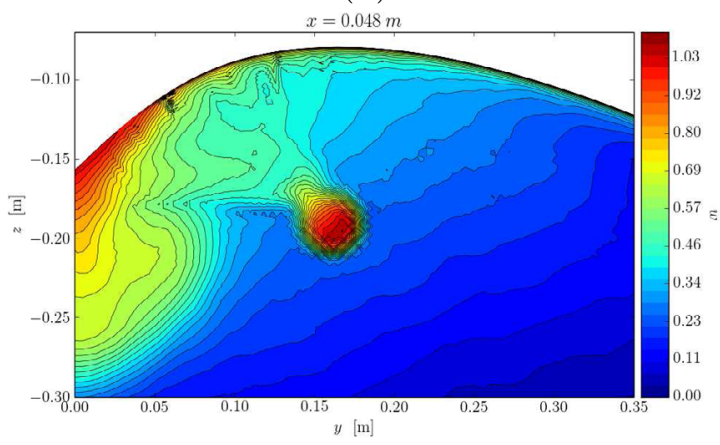

(d)

Figure 5: Comparison of the wake obtained on the different grids using different methods to experiment: (a) - experiment, (b) - SST-RANS on the fine mesh (c) - SST-IDDES on the coarse mesh, (d) - SST-IDDES on the fine mesh

Nevertheless, the predictions of the hybrid model are in close agreement with RANS for the mean velocity field, which shows, that no negative effects, related to modeled stress depletion [8] are present. At the same time the solution, obtained from the hybrid model is fully unsteady and contains the coherent vortical structures (see Figure 3). This allows for evaluation of the effect of the unsteadiness of the wake on the propeller, which will be conducted in the following 
sections. From this point of view it is interesting to see, how the mesh resolution influences the standard deviation of the longitudinal velocity field, $\sigma_{u}=\frac{1}{\left|\mathbf{u}_{\infty}\right|} \sqrt{\frac{1}{n-1} \sum_{i=0}^{n}\left(u_{x, i}-\left\langle u_{x}\right\rangle\right)^{2}} \times 100 \%$. As can be seen in Figure 7, $\sigma_{u}$ is quite sensitive to the mesh resolution in some points $(\mathrm{y}=0$, $\mathrm{y}=0.08)$, however, in the most of the considered points the variations between the meshes are less than $1 \%$.

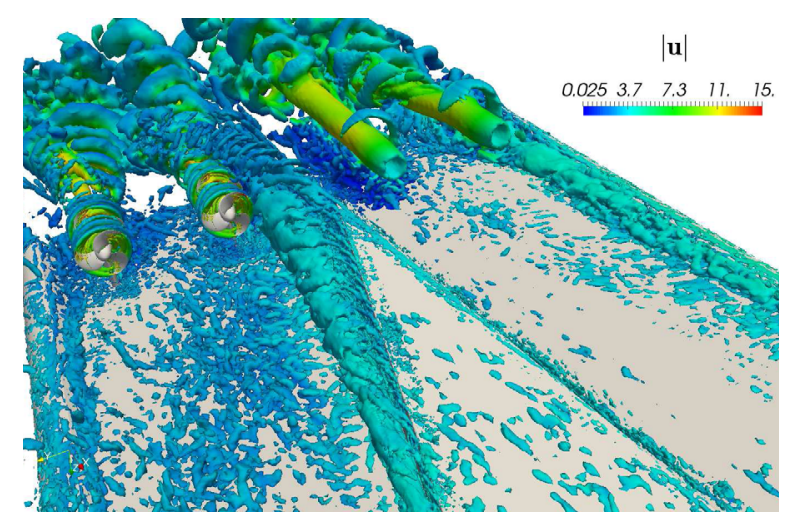

Figure 6: Vortical structures revealed in the wake of the river cruiser using $\lambda_{2}$ criterion. Motion with the drift angle $\beta=10^{\circ}, h / T=1.5$. With propellers.

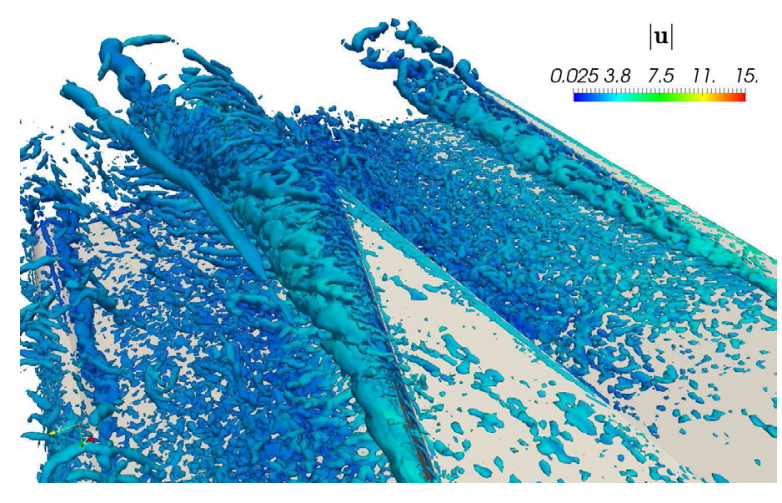

Figure 7: Vortical structures revealed in the wake of the river cruiser using $\lambda_{2}$ criterion. Motion with the drift angle $\beta=10^{\circ}, h / T=1.5$. Without propellers.

From the conducted validation study in was concluded, that the implementation of the SSTIDDES model works well for the considered flow: it provides almost the same time-averaged wake fraction as RANS, which are close to experimental data, while containing the unsteady vortical structures.

\section{ANALYSIS OF THE WAKE OF A RIVER CRUISER}

\subsection{Nominal wake}

First step of the application of the previously validated computational method was the analysis of the nominal wake of a river cruiser at full scale. The ship $\left(L_{w l}=135 \mathrm{~m}, B=12 \mathrm{~m}, T=\right.$ $2 \mathrm{~m})$ was considered moving in a shallow channel with $h / T=1.25,1.5,2.0$ at a speed of $3 \mathrm{~m} / \mathrm{s}$ with the drift angles $\beta=0^{\circ}, 10^{\circ}$.

Total number of cells used in computations was 20-22M depending on the depth. Stern region was filled with uniform isotropic mesh with the cell sizes $0.04 \times 0.04 \times 0.04 \mathrm{~m}^{3}$ in order to improve the performance of the hybrid method in the region of interest. As in the validation studies, the turbulence generator was applied upstream from the stern.

The ship has two azimuth thrusters installed on each side (see Figure 6). A thruster near the skeg will be in the following referred to as the inner (first) and the one near the bilge - as outer (or second). In the nominal wake computations the analysis concentrated on the evaluation of the wake nonuniformity and instationarity, which can contribute to the unsteady loadings on the propellers.

From the Figure 8 one can draw a number of important conclusions. Firstly, the wake in the disc of the outer thruster is much lower in all cases and is almost insensitive to the fairway 


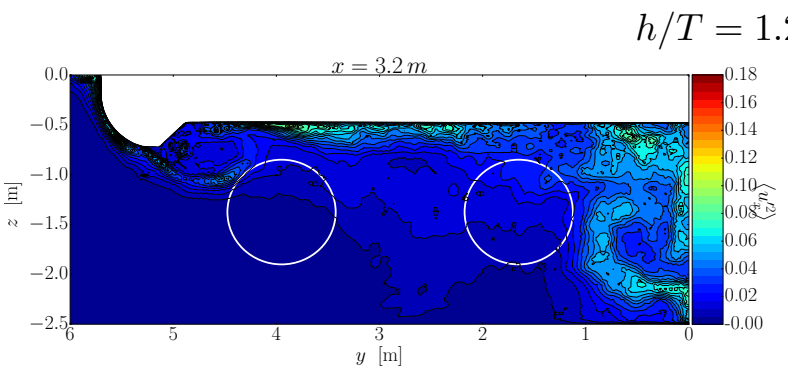

(a)

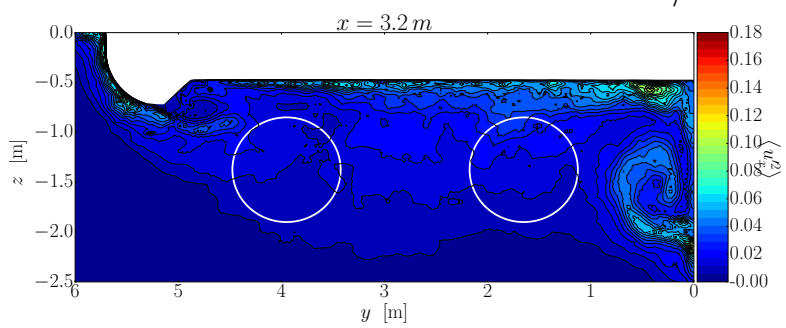

(c)

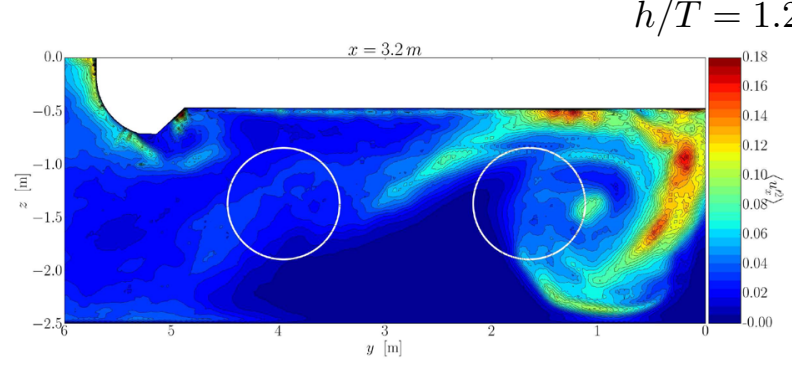

(e)

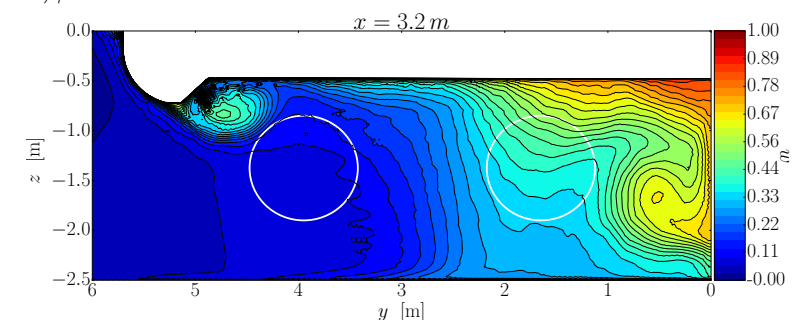

(b)

$0, \beta=0^{\circ}$

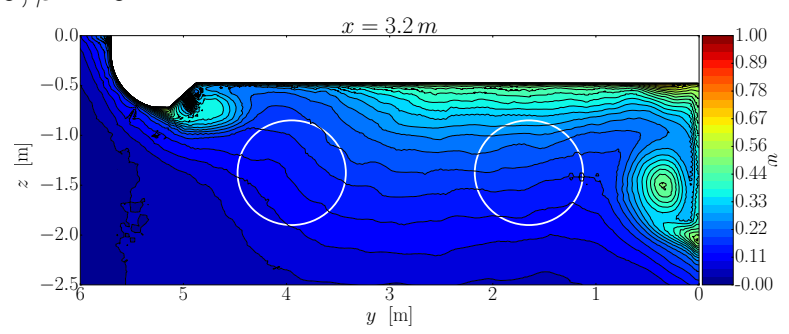

(d)

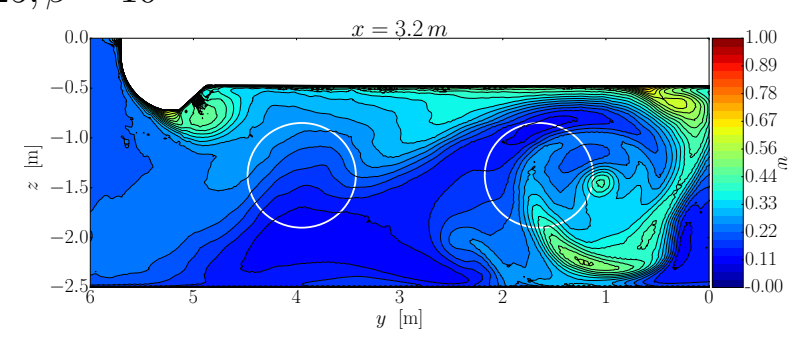

(f)

Figure 8: Plots of the resolved Reynolds stresses $\left\langle u_{x}^{\prime 2}\right\rangle$ (left) and wake (right).

depth. For the inner one this is not the case - the depth reduction leads to the rapid increase of the wake. Secondly, when the depth is reduced the velocity fluctuations tend to be concentrated near the inner thruster, whereas the outer one works in an almost undisturbed velocity field. No strong separations were observed at the stern at straight course.

The flow picture changes considerably, when the motion with the drift angle $\beta=10^{\circ}$ is considered. In this case the skeg vortex becomes very strong at smaller depths. The level of velocity fluctuations at the inner thruster increases, the amplitude of fluctuations reaches up to $15-20 \%$ of the inflow value. The wake is on the first pod is decreased, but at the second one considerably grows and in addition the irregularity of the velocity field is much stronger.

\subsection{Working thrusters}

After the analysis of the nominal wake, simulations with the rotating propellers were conducted for the same conditions, as listed above. Only the propellers form the port side were geometrically resolved, whereas the ones on the starboard side were replaced by the actuator 
disks, producing the prescribed thrust (see Figure 6). As the analysis showed, the wake and the resolved turbulence kinetic energy are very small on the starboard side and are very unlikely to cause any unsteady effects on the thrust.

Based on the propeller forces history, obtained during the simulations, the statistical analysis of thrust fluctuations was performed. The values of the maximum amplitude of fluctuations and their standard deviation $\sigma_{T}$ are shown in Tables 2 and 3.

Table 2: Statistics of the total thrust oscillations on the inner (1) and outer (2) pods for the motion at straight course

\begin{tabular}{lrrrrrr}
\hline$h / T$ & & 1.25 & & 1.5 & & 2.0 \\
\hline Pod & 1 & 2 & 1 & 2 & 1 & 2 \\
\hline$T_{\max }^{\prime} /\langle T\rangle \times 100[\%]$ & 5.78 & 5.07 & 5.25 & 5.19 & 5.05 & 6.55 \\
$\sigma_{T} \times 100[\%]$ & 2.44 & 2.07 & 2.26 & 2.42 & 2.20 & 2.86 \\
\hline
\end{tabular}

Table 3: Statistics of the total thrust oscillations on the inner (1) and outer (2) pods for the motion at $\beta=10^{\circ}$

\begin{tabular}{lrrrrrr}
\hline$h / T$ & & 1.25 & & 1.5 & & 2.0 \\
\hline Pod & 1 & 2 & 1 & 2 & 1 & 2 \\
\hline$T_{\max }^{\prime} /\langle T\rangle \times 100[\%]$ & 4.05 & 5.15 & 3.33 & 4.63 & 3.79 & 5.53 \\
$\sigma_{T} \times 100[\%]$ & 1.34 & 2.08 & 0.90 & 1.96 & 1.20 & 2.12 \\
\hline
\end{tabular}

In the tables one can observe the following trends. For the straight course the maximum fluctuation amplitude and the standard deviation of the thrust increase on the first pod as the depth gets smaller. On the outer pod reverse trend is observed. This means, that when the depth is decreased, the thrust fluctuations tend to be more intense of the inner pod. Motion with the drift angle surprisingly led to reduction of both statistical parameters on the first and on the second pod. This can be explained by the fact, that when the ship is moving with the drift angle, the turbulent structures, generated on the hull follow the flow direction and therefore are not transported directly into the propeller disc.

Analysis of the pressure fluctuations on the hull above the propellers revealed, that their amplitude is increasing from $3 \mathrm{kPa}$ to $7 \mathrm{kPa}\left(\right.$ for $\beta=0^{\circ}$ ) and to $8 \mathrm{kPa}\left(\right.$ for $\beta=10^{\circ}$ ) as the $h / T$ ratio decreases from 2.0 to 1.25 . This effect is pronounced for both propellers equally.

Even though the present research is concerned mostly with the unsteady thrust oscillations, the fact, that the cavitation may play a major role for the pressure oscillations on the hull (and the vibration) is acknowledged. Therefore, in order to evaluate, whether the cavitation can occur, an a-posteriori analysis of the cavitation inception regions was conducted. The regions, on the propeller blades, where the pressure is smaller than the vapour pressure $p_{v}$ are shown in Figure 9 for different motion conditions. There are two most important trends, which can be noticed. Firstly, the size of the regions $p<p_{v}$ decreases as the $h / T$ ratio gets smaller. However, in this case these regions are equally large on all blades. Secondly, when the motion with $\beta=10^{\circ}$ 
is considered, a significant difference in the size of low pressure zones on different blades can be observed. This effect stems from the fact, that the propeller works in an oblique flow, where the effective angle of attack depends on the blade position. Dramatic change of cavitation inception regions depending on the blade position allows to conclude that collapsing of cavitation sheets is likely to occur. This scenario of vibration intensification is described in [7].

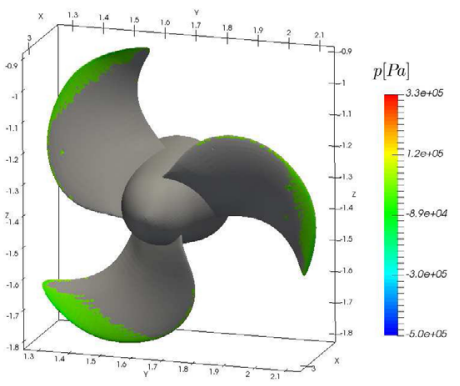

(a): $h / T=1.25, \beta=0^{\circ}$

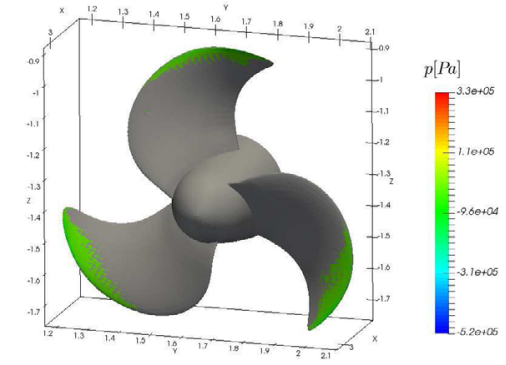

(b): $h / T=1.5, \beta=0^{\circ}$

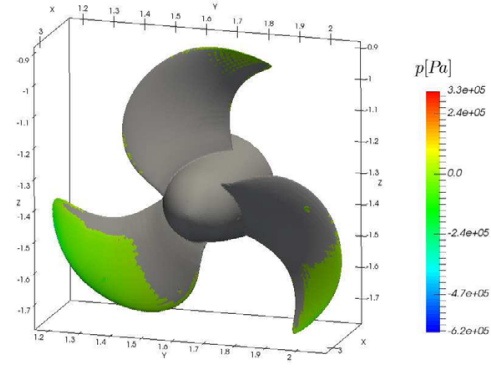

(c): $h / T=1.25, \beta=10^{\circ}$

Figure 9: Cavitation inception regions on the front propeller of the inner thruster for different motion conditions.

\section{CONCLUSIONS}

From the presented computational results, one can conclude, that the unsteady effects at the stern of cruise vessels are intensified, as the depth-to-draft ratio decreases. For the nominal wake, strong velocity oscillations and wake irregularities were observed at small depths. Simulations with the rotating propellers revealed the growth of the maximum fluctuation amplitude and the standard deviation of thrust in response to depth reduction (for inner pod at straight course and for both pods at $\beta=10^{\circ}$ ). The amplitude of the pressure pulses on the hull increases from $3 \mathrm{kPa}$ at $h / T=2.0$ to $7-8 \mathrm{kPa}$ at $h / T=1.25$. Finally, it was shown that the cavitation may occur, which would contribute to the pressure pulses on the hull plating. All of these factors can contribute to vibration intensification and therefore have to be accounted for during the design process of inland cruise vessels.

\section{ACKNOWLEDGEMENTS}

The support of the Mecklenburg State Graduate Foundation and the German Research Foundation (Deutsche Forschungsgemeinschaft) under the grant INST 264/113-1 FUGG is gratefully acknowledged. The authors would like to thank Mr. Benjamin Friedhoff and Prof. Bettar el Moctar from the Duisburg Development Centre for Ship Technology and Transport Systems (DST) for access to validation data and many helpful discussions.

\section{REFERENCES}

[1] D. Y. Adamian and A. K. Travin. Assessment of an approach to generating inflow synthetic turbulence for large eddy simulations of complex turbulent flows. Progress in Flight Physics, 
$5: 43-54,2013$.

[2] J. Binns, R. Brown, and N. Bose. Proceedings Of The Third International Symposium on Marine Propulsors. Australian Maritime College, University of Tasmania, 2013.

[3] J. Fröhlich and D. von Terzi. Hybrid LES/RANS Methods for the Simulation of Turbulent Flows. Progress in Aerospace Sciences, 44:349-377, 2008.

[4] M. Gritskevich, A. Garbaruk, J. Schütze, and F. Menter. Development of DDES and IDDES Formulations for the k- $\omega$ Shear Stress Transport Model. Flow, Turbulence and Combustion, 88(3):431-449, 2011.

[5] L. Larsson, F. Stern, and M. Visonneau. Gothenburg 2010 - a workshop on numerical ship hydrodynamics. In Proceedings. vol. 2. Department of Naval Architecture and Ocean Engineering, Chalmers University of Technology, 2010.

[6] S. List, K. Rügner, and B. Friedhoff. WAKE. An veränderliche Wassertiefen angepasste Konzepte zur Energiesparung durch Vergleichmäßigung des Propellerzustroms. Bericht 2162., 2015 .

[7] F. E. Reed and N. L. Bassett. Further Studies of Propeller-Excited Vibration on Great Lakes Cargo Ships. SNAME Transactions, 97:375-396, 1989.

[8] P. R. Spalart, S. Deck, M. L. Shur, K. D. Squires, M. Kh. Strelets, and A. Travin. A New Version of Detached-eddy Simulation, Resistant to Ambiguous Grid Densities. Theoretical and Computational Fluid Dynamics, 20(3):181-195, 2006.

[9] W. S. Vorus and J. R. Paulling. Vibration. The Principles of Naval Architecture Series. Society of Naval Architects and Marine Engineers, 2010. 